\title{
$\mathrm{SiC}$ 和 $\mathrm{B}_{4} \mathrm{C}$ 防弹插板抗多发弹打击损伤特性研究
}

\author{
崔凤单 ${ }^{1,2}$, 马 天 $^{2}$, 李伟萍 ${ }^{2}$, 吴国清 ${ }^{1}$
}

(1. 北京航空航天大学 材料科学与工程学院, 北京 100191；2. 军委后勤保障部 军需装备研究所, 北京 100082)

摘 要: 使用 87 式 $5.8 \mathrm{~mm}$ 钢芯弹分别对 $\mathrm{SiC}$ 和 $\mathrm{B}_{4} \mathrm{C}$ 复合防弹插板进行实弹靶试试验, 通过对鉴证靶凹陷深度、防 弹插板背凸体积和 $\mathrm{X}$ 射线数字直接成像检测系统(DR)对防弹插板弹击损伤情况进行分析，同时结合陶瓷材料显微 结构和力学性能分析对防弹插板抗多发弹打击损伤特性进行了研究。结果表明, $\mathrm{SiC}$ 和 $\mathrm{B}_{4} \mathrm{C}$ 防弹插板都能有效防御 3 发 $5.8 \mathrm{~mm}$ 钢芯弹的连续打击, 具有较好的抗多发弹打击性能; $\mathrm{B}_{4} \mathrm{C}$ 防弹插板与 $\mathrm{SiC}$ 防弹插板受弹击后鉴证靶凹 陷深度相当, 其背凸体积较 SiC 防弹插板降低超过 35\%, 陶瓷雉底面平均直径增加 30\%以上, 吸收了更多的弹丸冲 击动能, 这与 $\mathrm{B}_{4} \mathrm{C}$ 陶瓷具有较高的硬度有关。

关 键 词：防弹插板；多发弹打击；损伤特性；硬度

中图分类号: TJ04 文献标识码: A

\section{Damage Characteristics of $\mathrm{SiC}$ and $\mathrm{B}_{4} \mathrm{C}$ Ballistic Insert Plates Subjected to Multi-hit}

\author{
CUI Feng-Dan ${ }^{1,2}$, MA Tian², LI Wei-Ping ${ }^{2}$, WU Guo-Qing ${ }^{1}$
}

(1. School of Materials Science and Engineering, Beihang University, Beijing 100191, China; 2. The Quartermaster Research Institute, Logistic Support Department of China Central Military Commission, Beijing 100082, China)

\begin{abstract}
The ballistic impact tests of $\mathrm{SiC}$ and $\mathrm{B}_{4} \mathrm{C}$ ballistic insert plates were conducted with 87-type $5.8 \mathrm{~mm}$ steel core projectile. Through the analysis on the depth of penetration in clay witness, bulge volume at the back of the ballistic insert plates, and impact damage being nondestructively tested by digital radiograph (DR), the damage characteristics of $\mathrm{SiC}$ and $\mathrm{B}_{4} \mathrm{C}$ ballistic insert plates after multi-hit were investigated. Meanwhile, the microstructures and mechanical properties of ceramic materials were also studied. The results show that $\mathrm{SiC}$ and $\mathrm{B}_{4} \mathrm{C}$ ballistic insert plates effectively resist 87-type $5.8 \mathrm{~mm}$ steel core projectile for 3 times and show good multi-hit capability. The depths of penetration in clay witness of $\mathrm{B}_{4} \mathrm{C}$ ballistic insert plate are comparable with SiC ballistic insert plate. Compared with SiC ballistic insert plate, the volume of bulge at the back of $\mathrm{B}_{4} \mathrm{C}$ ballistic insert plate is reduced by more than $35 \%$, and the average diameter of the ceramic cone of $\mathrm{B}_{4} \mathrm{C}$ ballistic insert plate is increased by more than $30 \%$. The impact energy of the projectile is effectively absorbed by the $\mathrm{B}_{4} \mathrm{C}$ ballistic insert plate, which attributes to the high hardness of $\mathrm{B}_{4} \mathrm{C}$ ceramic.
\end{abstract}

Key words: ballistic insert plate; multi-hit; damage characteristics; hardness

碳化硅 $(\mathrm{SiC})$ 和碳化嗍 $\left(\mathrm{B}_{4} \mathrm{C}\right)$ 陶瓷复合装甲与传 统均质装甲(如钢装甲、铝合金装甲、钛合金装甲等) 相比, 具有质量轻、厚度薄、可设计性强等优点 ${ }^{[1-2]}$,
在防弹插板和武装直升机防护领域具有很大的应用 前景 ${ }^{[3]}$ 。在实战环境下，轻型装甲主要面临普通钢芯 弹，小口径穿甲弹和高速破片弹多发打击的威胁 ${ }^{[4]}$,

收稿日期：2016-12-02; 收到修改稿日期：2017-01-09

基金项目：国家基础研究计划 973 项目(613307)

National Basic Research Program of China(973 Program) (613307)

作者简介：崔凤单(1991-), 女，硕士研究生. E-mail:cuifd1991@163.com

通讯作者：吴国清, 副教授. E-mail: guoqingwu@buaa.edu.cn; 马 天, 高级工程师. E-mail: matian00@mails.tsinghua.edu.cn 
但陶瓷板材脆性较大，中弹后容易整体破碎，难以 抵抗多发子弹打击 ${ }^{[5]}$, 防弹插板抗多发弹打击问题 不容忽视。

目前, 陶瓷复合防弹插板抗多发弹打击性能的 研究主要集中在防弹插板结构的研究。孔晓鹏等 ${ }^{[6]}$ 提出了多发弹打击研究的 “几何间隔” 模拟方法, 分 析了粘结层材料和厚度对陶瓷/铝合金复合装甲抗 多发弹打击性能的影响。Wang 等 ${ }^{[7]}$ 提出一种具有抗 多发弹打击性能的混杂结构, 并对每层的防护机理 进行了分析。此外, 防弹插板抗多发弹打击损伤特 性与陶瓷材料微观结构和力学性能密切相关 ${ }^{[8]}$ 。

Savio 等 ${ }^{[9]}$ 认为陶瓷材料的断裂韧性显著影响陶瓷 板受弹丸撞击后的碎裂过程。Liu 等 ${ }^{[10]}$ 发现陶瓷材 料硬度越高, 子弹被粉碎成细小碎块的程度就越 大。Ray 等 ${ }^{[11]}$ 通过六种 $\mathrm{SiC}$ 基陶瓷的残余穿深试验 发现, 弹丸在背板中的穿透深度与陶瓷硬度存在明 显的负相关性。目前对防弹插板抗多发弹打击损伤 特性的研究相对较少, 而其损伤特性与使用者的安 全密切相关, 因此研究防弹插板抗多发弹打击损伤 特性具有重要的现实意义。

此外, 在水面作战的条件下, 水分子由于毛细 作用会逐渐进入复合材料的界面 ${ }^{[12]}$, 水解过程会破 坏聚酯中的不饱和基 ${ }^{[13-16]}$, 使纤维与树脂界面产生 分层现象 ${ }^{[17-18]}$, 因此水分子作用对防弹插板的影响 不容忽视。

本工作将 $\mathrm{SiC}$ 和 $\mathrm{B}_{4} \mathrm{C}$ 陶瓷分别与超高分子量聚 乙烯 (UHMWPE) 纤维层压板复合制备陶瓷复合 防弹插板, 经浸水处理后对其进行实弹靶试试验。 通过分析鉴证靶凹陷深度, 防弹插板背凸体积和防 弹插板弹着点周围的微裂纹及陶瓷破碎雉, 对浸水 条件下防弹插板经 3 发 87 式 $5.8 \mathrm{~mm}$ 钢芯弹打击后 的损伤特性进行系统研究。此外, 通过对陶瓷材料 进行密度、弯曲强度、弹性模量、维氏硬度和断裂 㓞性测试, 结合扫描电镜对陶瓷表面和断口的微观 形貌进行观察, 探讨了防弹插板抗多发弹打击损伤 特性与陶瓷力学性能的关系。

\section{1 实验方法}

\section{1 材料}

将制备好的 $\mathrm{SiC}$ 和 $\mathrm{B}_{4} \mathrm{C}$ 陶瓷板分别与一定厚度的 UHMWPE 纤维层压板进行复合, 施胶负压成防弹插 板。防弹插板尺寸为 $300 \mathrm{~mm} \times 250 \mathrm{~mm} \times 15 \mathrm{~mm}$, 其 中陶瓷板厚度 $5 \mathrm{~mm}$, UHMWPE 层压板厚度 $10 \mathrm{~mm}$ 。 在常温水槽中浸泡 $30 \mathrm{~min}$ 。

\section{2 实弹靶试试验}

以胶泥作为鉴证靶, 实弹靶试试验测试之前, 将防弹插板与软体防弹背心组合置于鉴证靶前面, 靶板设置按弹击方向依次为: 防弹插板、软体防弹背 心和鉴证靶，实弹靶试试验装置示意图如图 1 所示。 软体防弹背心为 UHMWPE 纤维, 面密度 $5.8 \mathrm{~kg} / \mathrm{m}^{2}$ 。 测试步骤按照《JXUB3010-2010木质陶瓷复合防弹 插板规范》, 采用 87 式 $5.8 \mathrm{~mm}$ 钢芯弹, 弹击着靶 速度为 $(920 \pm 10) \mathrm{m} / \mathrm{s}$ 。

\section{3 损伤检测}

实弹靶试试验测试后, 采用单尖头数显卡尺对 鉴证靶的凹陷深度进行测量。采用粘土材料对防弹 插板背弹面进行翻模, 构造模具, 再依次对模具中 的凹坑填砂，测量计算防弹插板经 3 发弹击后形成 的背凸体积。采用 NV-22C 型 X 射线数字直接成像 检测系统(DR)对防弹插板内部破坏情况进行无损 检测, 并采用 Digimizer 软件计算 3 发弹射击后的陶 瓷雉底面直径, 分别标记为 $D_{1} 、 D_{2}$ 和 $D_{3}$ 。

\section{4 表征方法}

采用感量为 $1.00 \mathrm{~g}$, 称量范围为 $0 \sim 5 \mathrm{~kg}$ 的衡量 器称量防弹插板质量 $M$, 采用点接触式三坐标测量 仪测量并采用计算机拟合造型的方法，计算防弹插 板面积 $S$, 按公式(1)计算防弹插板的面密度 $\rho$ 。

$$
\rho=\frac{M}{S}
$$

利用阿基米德法测量陶瓷样品的体积密度。采 用 AG-2000A 型材料试验机对样品的三点弯曲强度 和弹性模量进行测试, 测量跨距为 $30 \mathrm{~mm}$, 加载速 度为 $0.5 \mathrm{~mm} / \mathrm{min}$ 。采用 $\mathrm{AG}-2000 \mathrm{~A}$ 型材料试验机对 样品的断裂韧性进行测试, 采用单边切口梁法, 切 口为试样高度的 $1 / 3 \sim 1 / 2$, 切口宽度为 $0.2 \mathrm{~mm}$ 。采用 HVS-5 型 Vickevs 硬度仪对样品的硬度进行测试, 测 试载荷 $1 \mathrm{~kg}$, 加载时间 $15 \mathrm{~s}$ 。采用 Phenom ProX

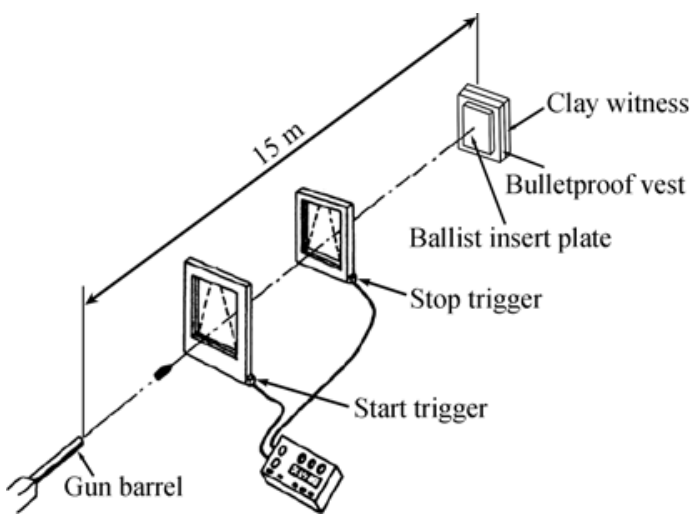

图 1 实弹靶试试验装置示意图 ${ }^{[18]}$

Fig. 1 Schematic diagram of ballistic test system ${ }^{[18]}$ 
型台式扫描电子显微镜对弹丸冲击前陶瓷样品经腐 蚀后的表面形貌和弹丸冲击后陶瓷样品的断口形貌 进行观察。

\section{2 结果与讨论}

\section{$2.1 \mathrm{SiC}$ 和 $\mathrm{B}_{4} \mathrm{C}$ 防弹插板抗多发弹打击损伤特 性分析}

$\mathrm{SiC}$ 和 $\mathrm{B}_{4} \mathrm{C}$ 防弹插板弹击后损伤结果如图 2 所 示, 可以看出, 在浸水条件下, 相同厚度的 $\mathrm{SiC}$ 和 $\mathrm{B}_{4} \mathrm{C}$ 陶瓷板与 UHMWPE 层压板复合后均未被穿 透，防弹插板整体外观受到的破坏较小，陶瓷面板 与背板之间没有出现明显的分层现象。表 1 为 $\mathrm{SiC}$ 和 $\mathrm{B}_{4} \mathrm{C}$ 防弹插板的实弹靶试试验结果, 3 次射击的 弹丸速度均在规定范围内, 两种防弹插板的鉴证靶 凹陷深度大致相同, 且均未超过 $25 \mathrm{~mm}$, 根据 《JXUB 3010-2010 木质陶瓷复合防弹插板规范》中 的规定, $\mathrm{SiC}$ 和 $\mathrm{B}_{4} \mathrm{C}$ 防弹插板在浸水条件下均符合 防弹要求。虽然 $\mathrm{B}_{4} \mathrm{C}$ 防弹插板与 $\mathrm{SiC}$ 防弹插板的鉴 证靶凹陷深度相当, 但其背凸体积与 $\mathrm{SiC}$ 防弹插板 相比降低了 $35.8 \%$, 说明 87 式 $5.8 \mathrm{~mm}$ 钢芯弹对 $\mathrm{B}_{4} \mathrm{C}$ 防弹插板造成的毁伤较小。此外, 防弹插板 的面密度计算结果表明, $\mathrm{B}_{4} \mathrm{C}$ 防弹插板的面密度 $\left(24.8 \mathrm{~kg} / \mathrm{m}^{2}\right)$ 比 $\mathrm{SiC}$ 防弹插板 $\left(28.0 \mathrm{~kg} / \mathrm{m}^{2}\right)$ 低 $11.4 \%$ 。 因此, $\mathrm{B}_{4} \mathrm{C}$ 防弹插板不仅可以提高士兵的作战灵活 度, 还可有效减少枪弹对士兵造成的损伤, 对个人 防护起到重要的作用。
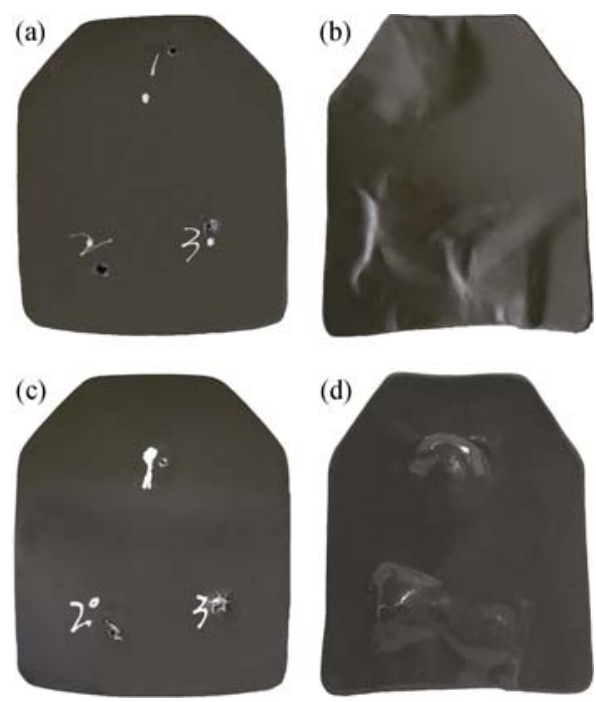

图 $2 \mathrm{SiC}$ 和 $\mathrm{B}_{4} \mathrm{C}$ 防弹插板迎弹面及背弹面损伤

Fig. 2 Morphologies of $\mathrm{SiC}$ and $\mathrm{B}_{4} \mathrm{C}$ ballistic insert plates after multi-hit

(a) Front face of $\mathrm{SiC}$ ballistic insert plate; (b) Black face of $\mathrm{SiC}$ ballistic insert plate; (c) Front face of $\mathrm{B}_{4} \mathrm{C}$ ballistic insert plate; (d) Black face of $\mathrm{B}_{4} \mathrm{C}$ ballistic insert plate
表 1 实弹靶试试验结果

Table 1 Results of ballistic test

\begin{tabular}{ccccc}
\hline $\begin{array}{c}\text { Ballistic } \\
\text { insert plates }\end{array}$ & $\begin{array}{c}\text { Ballistic } \\
\text { number }\end{array}$ & $\begin{array}{c}\text { Velocity } \\
/\left(\mathrm{m}^{-1} \mathrm{~s}^{-1}\right)\end{array}$ & $\begin{array}{c}\text { Depth of } \\
\text { indentation } \\
/ \mathrm{mm}\end{array}$ & $\begin{array}{c}\text { Volume } \\
\text { of bulge } \\
/ \mathrm{mm}^{3}\end{array}$ \\
\hline SiC/ & No.1 & 912 & 8.7 & 28320 \\
UHMWPE & No.2 & 921 & 9.4 & 38740 \\
& No.3 & 919 & 8.9 & 27070 \\
$\mathrm{~B}_{4} \mathrm{C} /$ & No.1 & 928 & 7.8 & 18570 \\
UHMWPE & No.2 & 910 & 6.3 & 17100 \\
& No.3 & 914 & 7.2 & 24110 \\
\hline
\end{tabular}

图 3 给出了浸水条件下 $\mathrm{SiC}$ 和 $\mathrm{B}_{4} \mathrm{C}$ 防弹插板 DR 检测图, 采用该检测系统可以清晰地观察到防 弹插板弹着点周围的裂纹和陶瓷破碎雉。SiC 和 $\mathrm{B}_{4} \mathrm{C}$ 防弹插板经首发射击后弹着点周围出现较多裂纹, 第 2 发和第 3 发弹丸射击时, 微裂纹开始扩展, 但 并未相互连接。第 1 发钢芯弹打击对防弹插板其他 区域造成的损伤并未严重降低其抗第 2 发和第 3 发 弹打击的能力。因此 $\mathrm{SiC}$ 和 $\mathrm{B}_{4} \mathrm{C}$ 防弹插板均可以防 御多发 78 式 $5.8 \mathrm{~mm}$ 钢芯弹的连续打击。

弹体冲击陶瓷面板后在弹体和陶瓷面板内同时 产生压缩应力波，应力波产生的应力大于陶瓷材料
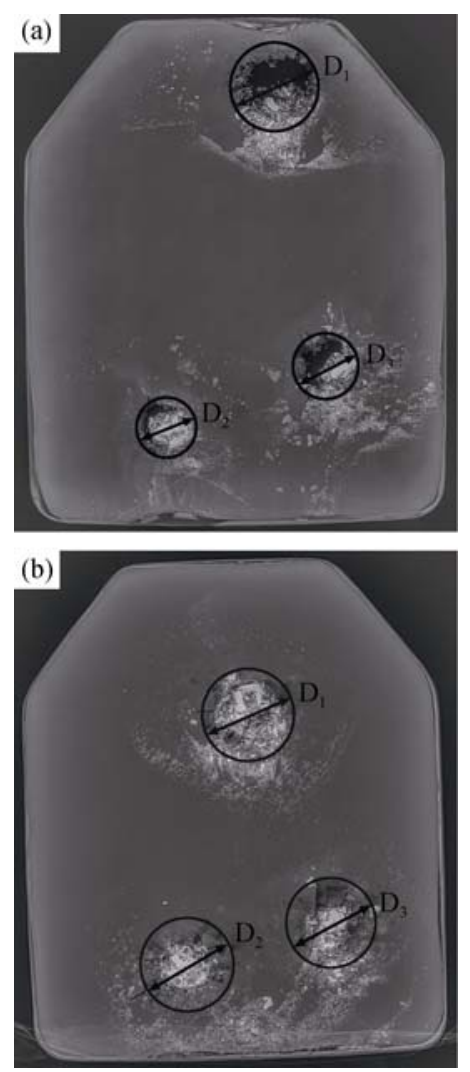

图 $3 \mathrm{SiC}$ 和 $\mathrm{B}_{4} \mathrm{C}$ 防弹插板 $\mathrm{DR}$ 检测图

Fig. 3 Digital radiograph of $\mathrm{SiC}$ and $\mathrm{B}_{4} \mathrm{C}$ ballistic insert plates after multi-hit

(a) $\mathrm{SiC}$ ballistic insert plat; (b) $\mathrm{B}_{4} \mathrm{C}$ ballistic insert plate 
的压缩强度，接触界面周围的陶瓷材料发生破坏; 当压缩应力波传播到陶瓷背面时，会反射拉伸应力 波，在拉伸应力波和周向应力的共同作用下，在陶 瓷面板中形成多条径向和环向的裂纹，最终形成陶 瓷破碎雉 ${ }^{[19]}$ 。在弹靶作用过程中，陶瓷破碎雉吸收 弹丸的冲击动能，传递冲击载荷，改变背板的破坏 形式，增大背板的破坏程度，是陶瓷复合防弹插板 的主要抗弹机理 ${ }^{[4]}$ 。 $\mathrm{SiC}$ 和 $\mathrm{B}_{4} \mathrm{C}$ 防弹插板经弹击后 形成的陶瓷雉底面直径测量结果见表 2, 可以看出, $\mathrm{B}_{4} \mathrm{C}$ 防弹插板比 $\mathrm{SiC}$ 防弹插板的陶瓷锥底面平均直 径大 $30.1 \%, \mathrm{~B}_{4} \mathrm{C}$ 防弹插板吸收了更多的弹丸冲击动 能, 这再次表明 $\mathrm{B}_{4} \mathrm{C}$ 防弹插板的抗多发弹打击性能 优于 $\mathrm{SiC}$ 防弹插板。

\section{$2.2 \mathrm{SiC}$ 和 $\mathrm{B}_{4} \mathrm{C}$ 装甲陶瓷的显微结构和力学性} 能分析

对比 $\mathrm{SiC}$ 和 $\mathrm{B}_{4} \mathrm{C}$ 防弹插板的实弹靶试试验结果

表 $2 \mathrm{SiC}$ 和 $\mathrm{B}_{4} \mathrm{C}$ 防弹插板陶瓷锥底面直径测量结果

Table 2 Diameter of the ceramic cone for $\mathrm{SiC}$ and $\mathrm{B}_{4} \mathrm{C}$ ballistic insert plates

\begin{tabular}{cccc}
\hline & $D_{1} / \mathrm{mm}$ & $D_{2} / \mathrm{mm}$ & $D_{3} / \mathrm{mm}$ \\
\hline SiC/UHMWPE & 54.2 & 35.6 & 41.8 \\
B 4 C/UHMWPE & 57.6 & 59.1 & 54.5 \\
\hline
\end{tabular}
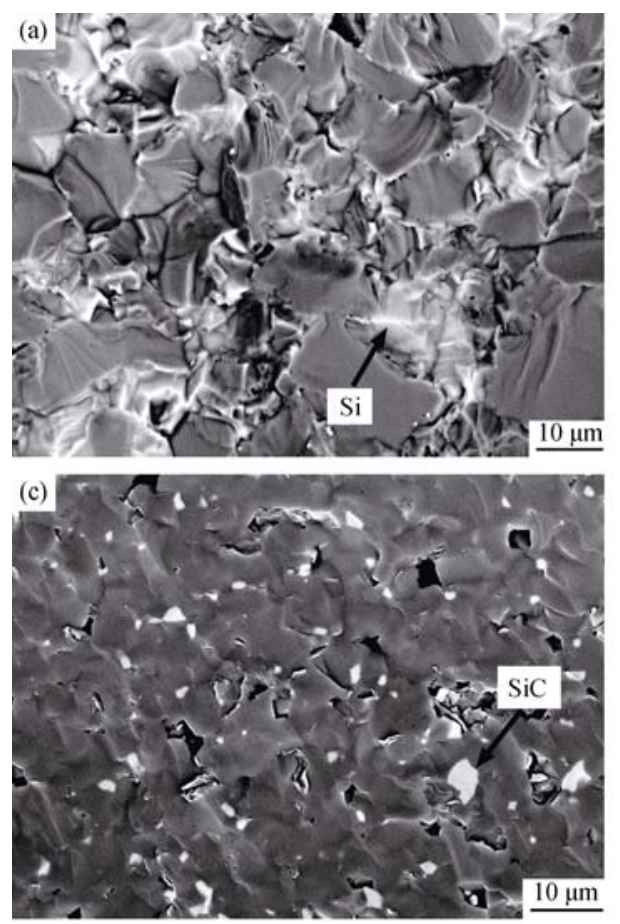

及 $\mathrm{DR}$ 检测结果可知，不同类型陶瓷作面板时，防 弹插板抗多发弹打击损伤特性有很大差别，这与陶 瓷材料的显微结构和力学性能密不可分。

图 4 为 $\mathrm{SiC}$ 和 $\mathrm{B}_{4} \mathrm{C}$ 陶瓷经弹丸冲击后的断口形 貌和 EDS 分析结果。从图 4(a)可以看出, SiC 烧结 体结构致密，气孔较少。材料的断裂方式以穿晶断 裂为主, 并伴随有少量沿晶断裂, 断口起伏较大, 使裂纹扩展更加曲折。EDS 能谱(图 4(b))显示白色 相为游离硅, 从其在弹丸冲击前 $\mathrm{SiC}$ 陶瓷的表面形 貌中也可观察到(图 5(a)), 这是由反应烧结 SiC 的烧 结机制决定的。已有研究表明 ${ }^{[20-21]}$, 游离硅的存在 会影响反应烧结 SiC 陶瓷的力学性能, 制备 SiC 陶 瓷应该尽量减小游离硅的尺寸和含量。图 4(c)呈现 出典型的 $\mathrm{B}_{4} \mathrm{C}$ 穿晶断裂的断口形貌，断面平滑，立 体感差，内部存在较多气孔，结合 EDS 能谱分析(图 4(d))发现第二相颗粒 $\mathrm{SiC}$ 均匀分布在 $\mathrm{B}_{4} \mathrm{C}$ 基体中, 并且在弹丸冲击前, $\mathrm{B}_{4} \mathrm{C}$ 陶瓷的表面形貌中也观察到 较多气孔和均匀分布的 SiC 颗粒(图 5(b))。第二相的 均匀分布有利于 $\mathrm{B}_{4} \mathrm{C}$ 烧结致密化及力学性能的提高。

$\mathrm{SiC}$ 和 $\mathrm{B}_{4} \mathrm{C}$ 陶瓷的密度、弹性模量、弯曲强度、 维氏硬度和断裂韧性如表 3 所示，从表中可以看出, 与 $\mathrm{SiC}$ 相比, $\mathrm{B}_{4} \mathrm{C}$ 的断裂韧性低, 但其弹性模量、弯
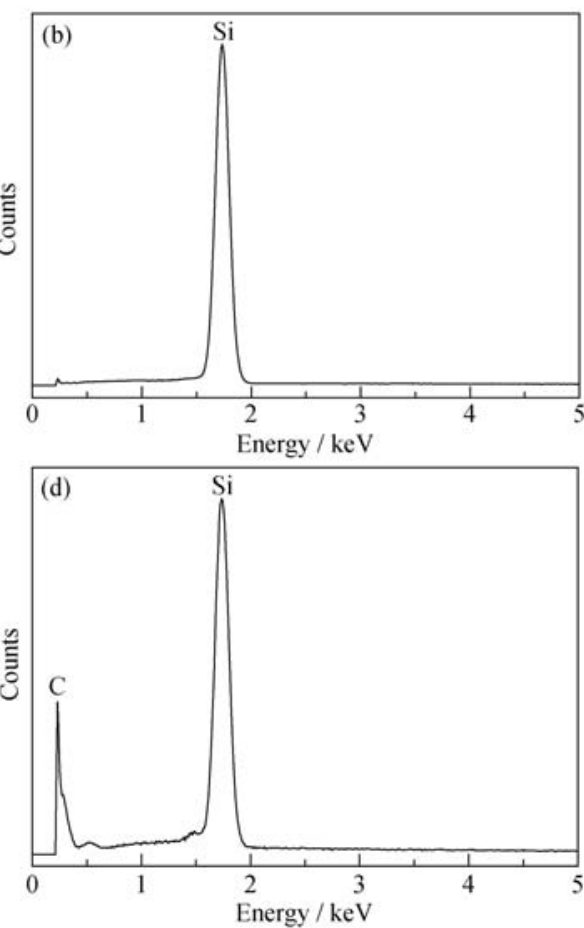

图 4 弹丸冲击后 $\mathrm{SiC}$ 和 $\mathrm{B}_{4} \mathrm{C}$ 陶瓷断口形貌的 SEM 照片和 EDS 图谱

Fig. 4 SEM morphologies of fracture surface and EDS patterns of $\mathrm{SiC}$ and $\mathrm{B}_{4} \mathrm{C}$ ceramic after impact (a) SEM of SiC ceramic; (b) EDS of Si particle in SiC ceramic; (c) SEM of $\mathrm{B}_{4} \mathrm{C}$ ceramic; (d) EDS of SiC particle in $\mathrm{B}_{4} \mathrm{C}$ ceramic 

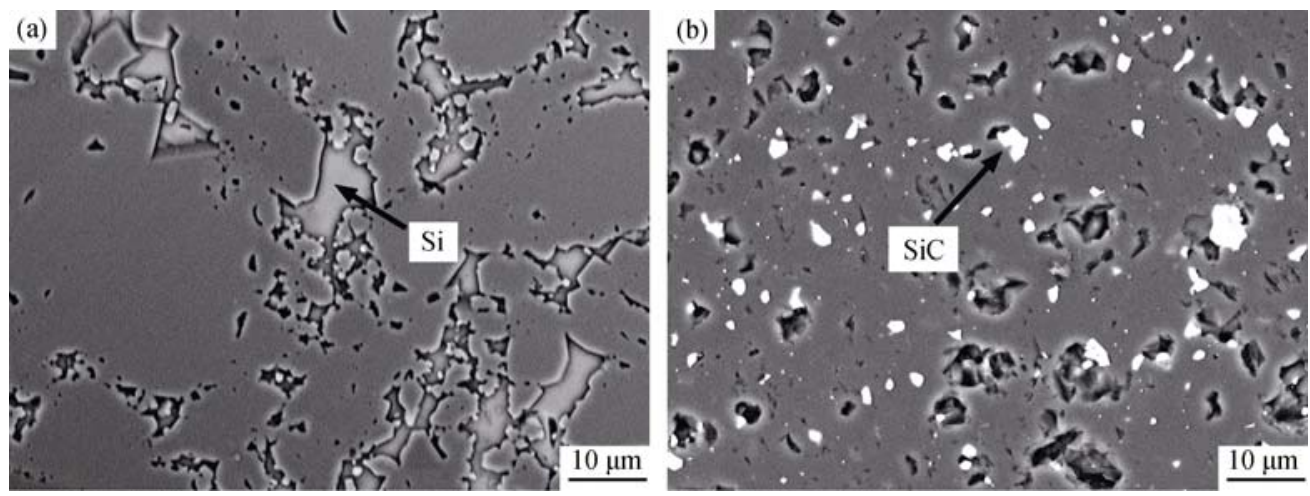

图 5 弹丸冲击前 $\mathrm{SiC}(\mathrm{a})$ 和 $\mathrm{B}_{4} \mathrm{C}$ (b)陶瓷表面形貌的 SEM 照片

Fig. 5 SEM morphologies of $\mathrm{SiC}(\mathrm{a})$ and $\mathrm{B}_{4} \mathrm{C}(\mathrm{b})$ ceramic before impact

表 $3 \mathrm{SiC}$ 和 $\mathrm{B}_{4} \mathrm{C}$ 装甲陶瓷的力学性能

Table 3 Property of $\mathrm{SiC}$ and $\mathrm{B}_{4} \mathrm{C}$ ballistic ceramics

\begin{tabular}{cccccc}
\hline Ceramics & $\begin{array}{c}\text { Density } \\
/\left(\mathrm{g} \cdot \mathrm{cm}^{-3}\right)\end{array}$ & $\begin{array}{c}\text { Elastic modulus } \\
/ \mathrm{GPa}\end{array}$ & $\begin{array}{c}\text { Flexural strength } \\
\text { /MPa }\end{array}$ & $\begin{array}{c}\text { Vickers hardness } \\
/ \mathrm{GPa}\end{array}$ & $\begin{array}{c}\text { Fracture toughness } \\
/\left(\mathrm{MPa} \cdot \mathrm{m}^{1 / 2}\right)\end{array}$ \\
\hline $\mathrm{SiC}$ & 3.07 & 418 & 445 & 22.9 & $3.6-4.0$ \\
$\mathrm{~B}_{4} \mathrm{C}$ & 2.50 & 466 & 543 & 33.4 & $2.8-3.0$ \\
\hline
\end{tabular}

曲强度和维氏硬度值分别高出 $11.5 \%$ 、 $22.0 \%$ 和 $45.8 \%$ ，这表明陶瓷材料的高硬度在提高防弹插板 防护能力方面发挥着重要作用。在冲击过程中，陶 瓷由于具有较高的硬度, 表面被碎裂成细小而坚 硬的碎块, 产生裂纹并形成陶瓷破碎雉。陶瓷材料 硬度越高，断裂韧性越低，弹丸冲击陶瓷时越易破 碎，陶瓷破碎雉体积越大，在侵彻过程中因磨损犁

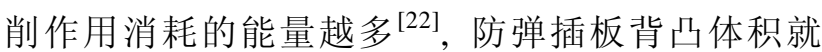
越小。弹丸冲击后, $\mathrm{B}_{4} \mathrm{C}$ 较高的硬度和低的断裂韧 性使其更容易碎裂，防弹插板的陶瓷雉底面直径 明显高于 $\mathrm{SiC}$ 防弹插板, 消耗了更多的弹丸能量, 背凸体积低于 $\mathrm{SiC}$ 防弹插板。这与理论分析的结果 也是一致的。

\section{3 结论}

本研究对 $\mathrm{SiC}$ 和 $\mathrm{B}_{4} \mathrm{C}$ 陶瓷复合防弹插板进行 实弹靶试试验，通过鉴证靶凹陷深度分析，防弹插 板背凸体积分析和防弹插板冲击损伤情况的观察, 并结合陶瓷材料的微观结构和力学性能对防弹插 板抗多发弹打击损伤特性进行的系统研究。主要结 论如下:

1) $\mathrm{SiC}$ 和 $\mathrm{B}_{4} \mathrm{C}$ 防弹插板都能防住 3 发 87 式 $5.8 \mathrm{~mm}$ 钢芯弹以 $920 \mathrm{~m} / \mathrm{s}$ 初速度的打击, 具有优良的抗多发
弹打击性能。

2) $\mathrm{B}_{4} \mathrm{C}$ 防弹插板与 $\mathrm{SiC}$ 防弹插板相比, 面密度降 低 $11.4 \%$, 鉴证靶凹陷深度相当, 其背凸体积较 SiC 防弹插板降低超过 35\%,陶瓷锥底面平均直径大 $30 \%$ 以上，呈现出更优的综合性能。

3) 从 $\mathrm{SiC}$ 和 $\mathrm{B}_{4} \mathrm{C}$ 的综合性能来看, $\mathrm{B}_{4} \mathrm{C}$ 陶瓷的 维氏硬度值高出 $45 \%$ 以上，其在冲击过程中更容易 碎裂, 吸收更多的弹丸冲击动能, 这可能是 $\mathrm{B}_{4} \mathrm{C}$ 防 弹插板表现出良好抗多发弹打击性能的主要原因。

\section{参考文献:}

[1] MOYNIHAN T J, CHOU S C, MIHALCIN A L. Application of the depth-of-penetration test methodology to characterize ceramics for personnel protection. ARL-TR-2119, 2000.

[2] KAUFMANN C, CRONIN D, WORSWICK M, et al. Influence of material properties on the ballistic performance of ceramics for personal body armour. Shock and Vibration, 2003, 10(1): 51-58.

[3] CLAYTON J D. Penetration resistance of armor ceramics: dimensional analysis and property correlations. International Journal of Impact Engineering, 2015, 85: 124-131.

[4] JIANG Z G, ZENG S Y, SHEN Z Q. Research progress on lightweight ceramic armor structure. Acta Armamentarii, 2010, 31(5): 603-610.

[5] KARANDIKAR P, GIVENS B, LISZKIEWICZ A. Effects of novel geometric designs on the bllistic performance of ceramics. 
Advances in Ceramic Armor X, 2015, 4: 13-22.

[6] KONG X P, JIANG Z G, LIU F. Simulation on ceramic composite armors against multi-hit of APPs. Applied Mechanics \& Materials, 2011, 105: 1648-1652.

[7] WANG Q, CHEN Z, CHEN Z. Design and characteristics of hybrid composite armor subjected to projectile impact. Materials \& Design, 2013, 46(4): 634-639.

[8] MEDVEDOVSKI E. Ballistic performance of armour ceramics: Influence of design and structure. Part 2. Ceramics International, 2010, 36(7): 2117-2127.

[9] SAVIO, S G, MADHU, V, GOGIA, A K. Ballistic performance of alumina and zirconia-toughened alumina against 7.62 armour piercing projectile. Defence Science Journal, 2014, 64(5): 464-470.

[10] LIU C Y, TUAN W H, CHEN S C. Ballistic performance of liquidphase sintered silicon carbide. Ceramics International, 2013, 39(7): 8253-8259.

[11] RAY D, FLINDERS R, ANDERSON A, et al. Effect of room temperature hardness and toughness on the ballistic performance of SiC-based ceramics. Ceramic Engineering and Science Proceedings, 2005, 26(7): 131-142.

[12] ZHANG J H, ZHAN M S. Visual experiments for water absorbing process of fiber-reinforced composites. Journal of Composite Materials, 2004, 38(6): 779-790.

[13] KOOTSOOKS A, MOURITZ A P. Seawater durability of glass-and carbon-polymer composites. Composites and Science Technology, 2004, 64(10): 1503-1511.

[14] SURI C, HAMADA H, KOYAMA K. Blister appearance in thermoplastic composites. Advanced Composite Materials, 2001, 10(1):
63-75.

[15] HODZIC A, KIM J K, LOWE A E, et al. The effects of water aging on the interphase region and interlaminar fracture toughness in polymer-glass composites. Composites Science and Technology, 2004, 64(13): 2185-2195.

[16] ALVAREZ V A, VAZQUEZ A. Effect of water sorption on the flexural properties of fully biodegradable composites. Journal of Composite Materials, 2004, 38(10): 1165-1181.

[17] IMIELINSKA K, GUILLAUMAT L. The effect of water immersion ageing on low-velocity impact behaviour of woven aramid-glass fibre/epoxy composites. Composites Science and Technology, 2004, 64(13): 2271-2278.

[18] 张建春, 张华鹏. 军用盔甲. 北京：长城出版社, 2003: 153, 162-163, 166-170.

[19] ZAERA R, SANCHEZ-GALVEZ V. Analytical modeling of normal and oblique ballistic impact on ceramic/metal lightweight armors. International Journal of Impact Engineering, 1998, 21(4): 133-148.

[20] CHAKRABARTI O P, GHOSH S, MUKERJI J. Influence of grain size, free silicon content and temperature on the strength and toughness of reaction-bonded silicon carbide. Ceramics International, 1994, 20(5): 283-286.

[21] WILHELM M, KORNFELD M, WRUSS W. Development of SiC-Si composites with fine-grained SiC microstructures. Journal of the European Ceramic Society, 1999, 19(12): 2155-2163.

[22] HUANG L Z, ZHANG A P, WANG Y. Study of wut mechanism and sharp by oneself affect of ball-proof ceramics. Journal of Changchun Institute of Optics and Fine Mechanics, 2002, 25(4): 1-3. 